\title{
La Teleodontología una Herramienta Fundamental en Tiempos de Pandemia y post COVID -19, su Utilidad en las Diferentes Especialidades Odontológicas
}

\author{
Teledentistry a Fundamental Tool in Times Pandemic and \\ Post COVID-19, its Usefulness in Different Dental Specialties
}

Michelle Morón Araújo

MORÓN, A. M. La teleodontología una herramienta fundamental en tiempos de pandemia y post COVID -19, su utilidad en las diferentes especialidades odontológicas. Int. J. Odontostomat., 15(1):43-50, 2021.

RESUMEN: La infección por Coronavirus ha sido considerada por la OMS (Organización Mundial de la Salud) como una pandemia global. Desde su origen ha puesto una amenaza para la salud mundial, no tanto por su sintomatología, sino por su rápida propagación, contagio y las complicaciones respiratorias que producen en pacientes con otras afecciones crónicas de base. La telesalud ha causado protagonismo en esta época de COVID-19 ya que muchas consultas presenciales han sido cerradas para evitar contagios y se ha preferido la virtualidad. La teleodontología no ha sido la excepción facilitando la consulta de pacientes, especialistas y/o proveedores de atención odontológica a distancia para proporcionar servicios odontológicos especialmente diagnósticos y preventivos optimizados en ausencia física del paciente. Este artículo de revisión de literatura tiene como objetivo describir el uso de la teleodontología como una herramienta en tiempos de COVID-19 su aplicación y uso en las diferentes especialidades odontológicas.

PALABRAS CLAVE: COVID-19, Tecnología, Teleodontología.

\section{INTRODUCCION}

En Diciembre del 2019 en la ciudad de Wuhan en China se identificó un nuevo corona virus 2019nCov como causante de infección respiratoria en personas que estuvieron en un mercado de animales salvajes incluyendo los murciélagos. Con propagación a nivel mundial se convirtió en una emergencia sanitaria potencial (Sigua-Rodríguez et al., 2020).

La telesalud es el conjunto de actividades que se llevan a cabo a distancia, en las áreas de promoción, prevención, diagnóstico, tratamiento o rehabilitación; realizadas por profesionales de la salud que utilizan tecnologías de la información y la comunicación (Almazrooaa et al., 2020).

La telesalud ha tomado protagonismo, esto debido a que el hecho de los centros de salud podrían ser fuentes de contagio, especialmente en un contexto donde no se encuentran implementos de bioseguridad. Existen modelos de atención que evi- tan el contacto cara a cara entre el profesional de salud y el paciente, especialmente por la atención remota a través de video llamada o teleconsulta, ya que evita el riesgo de contagio y reduce así la propagación del COVID-19 (Almazrooaa et al.)

Los sistemas de telemedicina se desarrollan para la computación generalizada. Una subcategoría de telemedicina es la teleodontología. Gracias a la teleodontología, es posible realizar consultas distantes, compartiendo información digital como imágenes, trabajo cooperativo, documentos, radiografías, etc. (Márquez, 2020).

\section{HISTORIA Y CONCEPTOS}

El avance de las ciencias de la salud en los últimos años ha estado ligado con el desarrollo en las tecnologías de comunicación, que han permitido sobrepasar situaciones adversas y hostiles, el heliógrafo 
el cual fue usado desde la peste bubónica en Europa como único medio de comunicación masivo para prevenir y advertir de la letalidad de dicha enfermedad. (Cáceres-Méndez et al., 2011; Avula, 2015).

El telégrafo en la guerra civil facilitó los pedidos de suministros médicos, el teléfono en el inicio del siglo XX, el cual fue acogido rápidamente por la población médica, la radio a partir de la primera guerra mundial y en los conflictos de Corea y Vietnam, donde la radio fue usada regularmente para informar el estado de salud de sus tropas y solicitar ayuda médica. (Cáceres-Méndez et al.).

La teleodontología es un subconjunto de telesalud que se está formando rápidamente, un campo que ya tiene un impacto considerable en la industria del cuidado de la salud. El término "teledentistry" fue utilizado en 1997 por Cook quien lo definió como la práctica de usar tecnologías de videoconferencia consejos sobre el tratamiento a distancia. Debido al enorme crecimiento de las capacidades tecnológicas, la teleodontología posee el potencial de cambiar fundamentalmente la práctica actual y la cara del cuidado dental (Cáceres-Méndez et al.).

En Boston el 1 de mayo de 2018, la American Teledentistry Association (ATDA) se lanzó en un esfuerzo por dar forma a la conversación en torno a la práctica de la teleodontología como una herramienta para aumentar el acceso a la atención de millones de estadounidenses mediante el uso de innovadores avances tecnológicos (American Teledentistry Association, 2018).

La ATDA define la teleodontología como: el uso de tecnologías electrónicas de información, imagen y comunicación, que incluyen comunicaciones interactivas de audio, video y datos, así como tecnologías de almacenamiento y reenvío, para proporcionar y apoyar la prestación, el diagnóstico, la consulta, la transferencia de información dental y la educación sobre la atención dental (American Teledentistry Association).

La telesalud no es un servicio específico; se refiere a una amplia variedad de tecnologías y tácticas para brindar servicios médicos, de salud y educativos virtuales. Como un término general, se define aún más cuando se aplica a disciplinas específicas de atención médica, como la odontología. La teleodontología, de acuerdo con la declaración de política integral de la ADA sobre la teleodontología, se refiere al uso de sistemas y metodologías de telesalud en odontología (Bradley et al., 2010).
La teleodontología puede incluir atención al paciente y entrega de educación utilizando, entre otras, las siguientes modalidades:

- Video en vivo (síncrono): interacción bidireccional en vivo entre una persona (paciente, cuidador o proveedor) y un proveedor que utiliza tecnología de telecomunicaciones audiovisuales interactivas (Cardwell, 2020, ADA, 2015). (Fig. 1.)

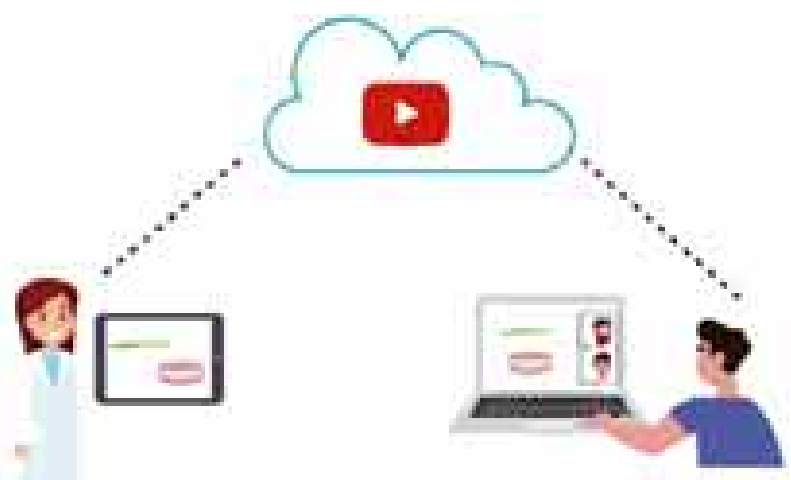

Fig. 1. Modalidad sincrónica entre paciente y odontólogo.

- Almacenamiento y reenvió (asíncrono): transmisión de información de salud registrada (por ejemplo, radiografías, fotografías, videos, impresiones digitales y fotomicrografías de pacientes) a través de un sistema de comunicaciones electrónicas seguro a un profesional, que utiliza la información para evaluar o diagnosticar la condición de un paciente o prestar un servicio (Cardwell; ADA, 2015) (Fig. 2).

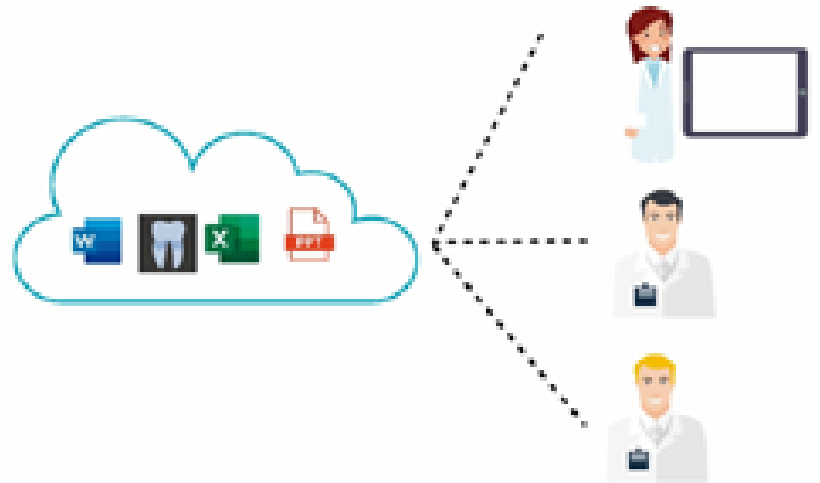

Fig. 2. Modalidad asincrónica entre paciente y odontólogo.

- Monitoreo remoto de pacientes (RPM): recopilación de datos médicos y de salud personal de un individuo en un solo lugar a través de tecnologías de comunicación electrónica, que se transmite a un proveedor (a veces a través de un servicio de procesamiento de datos) en una ubicación diferente para su uso en la atención y el soporte relacionado de la atención (Cardwell). (Fig. 3). 


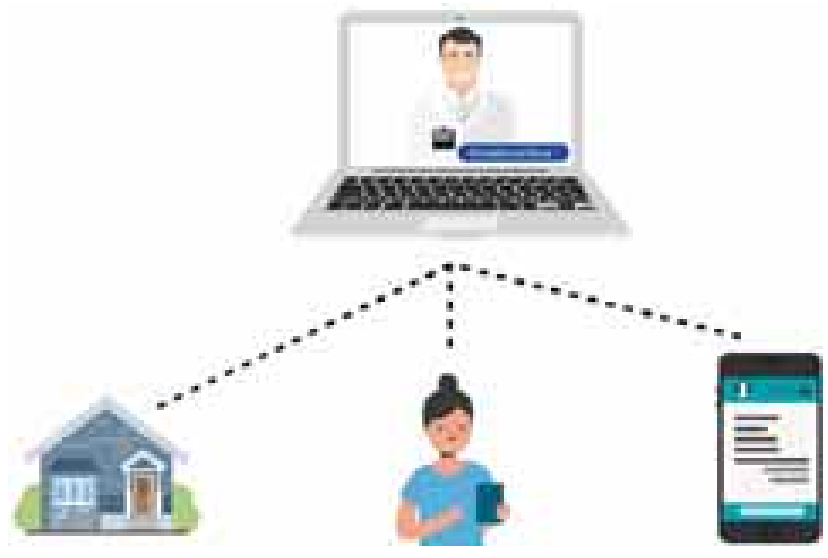

Fig. 3. Monitoreo remoto de pacientes.

- Salud móvil: Prácticas y educación de salud y salud pública respaldada por dispositivos de comunicación móvil y aplicaciones de software, incluidos teléfonos celulares, tabletas y asistentes digitales personales. (Bradley et al.; Cardwell 2020, ADA) (Fig. 4).
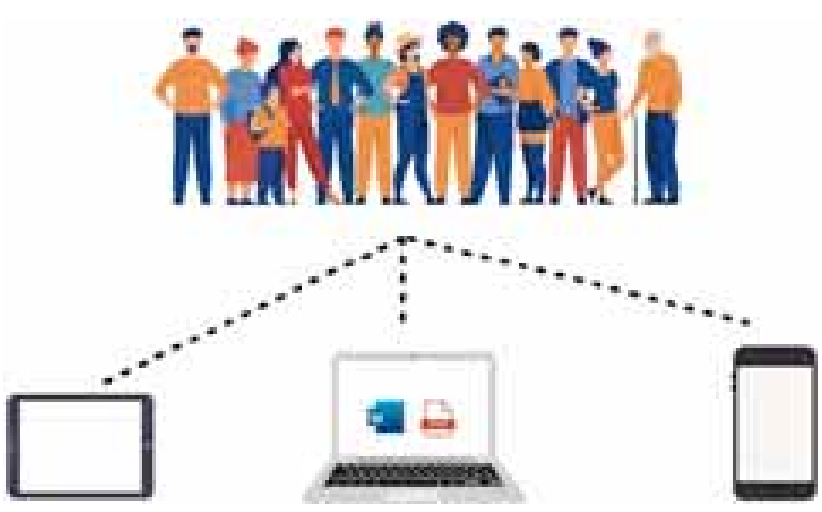

Fig. 4. Salud móvil.

\section{TELEODONTOLOGÍA EN SERVICIOS ODONTOLOGICOS}

\section{Teleconsulta y telediagnóstico}

Utilización de la tecnología de teleodontología para el diagnóstico de la caries dental ha tenido una creciente aceptación en los últimos años. El desarrollo de un método para reducir el tiempo empleado en la fotografía dental y para simplificar la transmisión, el procesamiento y la revisión de imágenes dentales que también proporcionarían el nivel de evaluación comparable al examen oral visual, aumentaría la adopción y aceptación del modelo de teleodontología móvil (Estai et al., 2016a,b).
La teleconsulta tiene como objetivo proporcionar consultas remotas a pacientes que asisten o que no pueden asistir presencialmente a servicios médicos u odontológicos. Un asistente y/o el profesional pueden estar presente durante la teleconsulta e interactuar con el paciente. El odontólogo recibe información pertinente (radiografía del área, una fotografía intraoral, toda la información de la historia clínica) de un paciente a través de internet evaluaría y sugeriría el plan de tratamiento adecuado (Rojas et al., 2015).

Una herramienta fácil, viable y accesible, útil tanto para profesionales de la salud como para pacientes. Esta comunicación podría ocurrir a través de aplicaciones de mensajería instantánea (WhatsApp, Telegram, Instagram, SMS, Messenger) y aplicaciones de video llamada (Google Meet, Skype, Facetime, WhatsApp).

En un estudio confirmaron el uso de WhatsApp como soporte en el diagnóstico oral, en el que el $82 \%$ de los casos teleconsultados coinciden con el diagnóstico clínico-patológico, lo que sugiere que es una buena opción para la teleodontología (Petruzzi et al., 2016; Telles et al., 2020).

\section{Teleeducación}

Teleeducación está ganando popularidad para complementar los métodos de enseñanza tradicionales en educación dental y proporcionar nuevas oportunidades para estudiantes de odontología y odontólogos. La educación formal en línea se puede dividir en dos categorías principales: autoinstrucción basada en la web y videoconferencia interactiva. El sistema educativo de autoinstrucción basado en la web contiene información que se ha desarrollado y almacenado antes de que el usuario acceda al programa (Rodríguez-Díaz \& Cabardillo, 2014; Ghai 2020).

Autoinstrucción basada en la web: La educación formal en línea se puede dividir en dos categorías principales: autoinstrucción basada en la web y videoconferencia interactiva. El sistema educativo de autoinstrucción basado en la Web contiene, información que se ha desarrollado y almacenado antes de que el usuario acceda al programa. La ventaja de la autoinstrucción basada en la web es que el usuario puede controlar el ritmo de aprendizaje y puede revisar el material tantas veces como lo desee (QuispeJuli et al., 2020).

Videoconferencia interactiva: Incluye una videoconferencia interactiva en vivo (con al menos una 
cámara configurada donde se transmite la información del paciente; sin embargo, las cámaras en ambos lugares son ideales) e información de apoyo (como historial médico del paciente, radiografías) que se pueden enviar antes o al mismo tiempo (por ejemplo, por email) como la videoconferencia (con o sin el paciente presente). La ventaja de este estilo educativo es que el usuario (generalmente el proveedor de atención médica del paciente) puede recibir retroalimentación (Estai et al., 2016a,b, 2018).

El mundo vive un fenómeno inesperado. Nunca se anticipó ni hubo planes para enfrentarlo, pero mientras se le hace frente a la pandemia del SARS-CoV-2 (COVID-19), muchos sectores han visto la necesidad de reinventarse y acudir a la innovación para seguir adelante con sus rutinas y compromisos, y el sector salud no es la excepción (Márquez; Turner et al., 2020).

Durante la epidemia del SARS (síndrome respiratorio agudo severo), en China se utilizaron iniciativas ingeniosas que condujeron al progreso en la educación médica. Se implementaron técnicas de aprendizaje en línea basado en problemas. Estos métodos resultaron populares y son aplicados hasta la actualidad de forma complementaria (Márquez 2020, QuispeJuli et al.).

Durante esta pandemia del Covid-19 muchas instituciones, universidades, federaciones etc., han realizado seminarios, cursos, congresos web (webinars, charlas, exposiciones, foros, debates) por medio de plataformas virtuales como ZOOM, Go to webinars, Microsoft Team y redes sociales como Instagram y Facebook entre otras, lo cual ha sido de gran importancia y de mucha utilidad como medio educativo de entrenamiento y actualización para profesionales en salud oral, estudiantes y pacientes en todo el mundo. (Fig. 5).

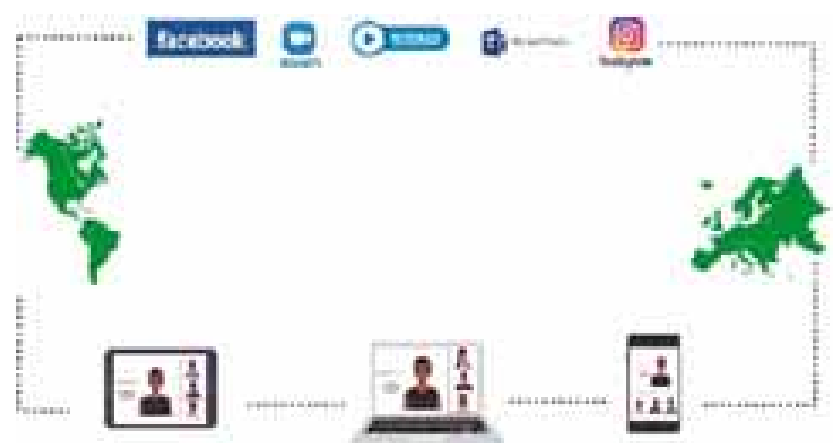

Fig. 5. Participación de instituciones, universidades, federaciones y entrega de contenidos durante la pandemia COVID19.
La pandemia de COVID-19 tiene serias implicaciones para la educación médica y de ciencias de la salud. Los estudiantes de las áreas en salud requieren exposición clínica, sin embargo, las rotaciones frecuentes dentro de los hospitales y clínicas convierten a los estudiantes de salud posibles vectores de COVID-19. Las facultades de odontología en las diferentes universidades del país y el mundo han suspendido sus actividades prácticas académicas y podría mantenerse por un periodo prolongado a medida que la pandemia de COVID-19 continúe desarrollándose, lo que ha llevado a los estudiantes de odontología a recibir clases virtuales y seguir con sus programas académicos sin realizar sus prácticas en pacientes (Meng et al., 2019; Telles et al.).

Muchos congresos, cursos y seminarios han sido suspendidos lo cual varios se han realizado gratuitamente y de manera virtual en las diferentes plataformas virtuales tratando diferentes temas de acuerdo a las diferentes especialidades odontológicas en diferentes países. Actualmente, la Organización Mundial de la Salud a través de su plataforma OpenWHO ofrece diferentes cursos, dentro de ellos el COVID19.

\section{TELEODONTOLOGÍA EN DIFERENTES ESPECIA- LIDADES ODONTOLOGICAS}

\section{Teleodontología en endodoncia}

Las lesiones periapicales son la patología más común que enfrentan los endodoncistas cualquier falla en el diagnóstico diferencial y el pronóstico del tratamiento de las lesiones periapicales puede causar complicaciones, problemas, pérdida de tiempo y dinero (Jain, 2016).

Los sistemas de tecnología moderna ayudan a buscar el asesoramiento de expertos a tiempo y a formular un plan de tratamiento. Con el uso de métodos de teleodontología, el diagnóstico de las lesiones periapicales se puede evaluar adecuadamente, y se puede diseñar un plan necesario para el tratamiento endodóntico adecuado de las lesiones (Jain; Cruvinel et al., 2019).

El método incluye información digital para cada diente de interés. Especialistas en endodoncia, son informados a través de sus teléfonos móviles sobre la solicitud recibida, después de lo cual descargan las imágenes digitales y los datos de anamnesis que las acompaña, establecen el diagnóstico y sugieren un 
tratamiento, luego publican esta información en un servidor en línea, que informa al odontólogo solicitante de la consulta sobre la respuesta recibida (Jain).

\section{Teleodontología en odontopediatría}

La Asociación Americana de Odontopediatría (ALOP) reporta de experiencias previas en las cuales el odontopediatra ha realizado diagnóstico de caries y recomendaciones de tratamiento con el uso de teleodontología, con la utilización de cámaras intraorales o imágenes obtenidas a través de teléfonos inteligentes (Asociación Latinoamericana de Odontopediatría).

La pre-consulta incluye la provisión de un cuestionario previo enviado por medios digitales, que puede ser diligenciado y enviado antes de la consulta sincrónica, o para apoyar la consulta asincrónica. Es recomendable tener una interacción previa con el paciente o sus cuidadores antes de la consulta que se pretende desarrollar, según las necesidades del paciente o las posibilidades de apoyo que el especialista pueda brindar (Asociación Latinoamericana de Odontopediatría).

Adicionalmente a ese cuestionario previo, se debe incluir una guía de consulta virtual que debe ser firmada bajo el acuerdo que al realizar la consulta se aceptaron las estipulaciones allí contenidas, incluyendo especificaciones sobre el alcance de la consulta virtual, las limitaciones y los pasos básicos durante esa consulta ya sea sincrónica o asincrónica (Asociación Latinoamericana de Odontopediatría).

Dependiendo del momento de la pandemia COVID-19 en cada región, y de las regulaciones establecidas por los organizamos estatales y de salud, la consulta odontológica se verá dirigida hacia la atención exclusiva de urgencias, la realización de tratamientos prioritarios, hasta la reapertura de la consulta para procedimientos de rutina (Asociación Latinoamericana de Odontopediatría; Purohit).

\section{Teleodontología en periodoncia}

La enfermedad periodontal, que es una enfermedad silenciosa de las estructuras subyacentes de los dientes, sigue siendo un problema dental global y es una de las razones más comunes de pérdida de dientes en poblaciones adultas. La detección y el diagnóstico rápidos pueden prolongar la vida de los dientes en la cavidad oral (Avula, 2015).
La videoconferencia en tiempo real permite a las personas en dos o más sitios comunicarse entre sí mediante una pantalla digital para mostrar una imagen de video de la persona o personas en uno o ambos sitios. Se utilizan una cámara de video y teléfonos con altavoz para mejorar la visibilidad y la audibilidad de las personas en ambos extremos. Por lo general, ambas partes acuerdan un horario de reunión y la información que se intercambia en tiempo real se transmite simultáneamente entre los sitios (Avula).

La terapia periodontal de apoyo se puede administrar a pacientes a través de distancias geográficas. Las visitas de mantenimiento periodontal también pueden reducirse enormemente al monitorearlas en vivo con la ayuda de la televigilancia. La televigilancia es una forma conveniente para controlar y realizar seguimiento la salud oral para que los pacientes eviten salir de sus casas (Avula).

\section{Teleodontología en ortodoncia}

El examen de la teleodontología para identificar la necesidad de tratamiento ortodontico resulta ser tan efectivo para exámenes clínicos. La teleodontología se ha utilizado en clínicas para evaluar la necesidad de ortodoncia y para proporcionar instrucciones a los pacientes que estén en un tratamiento de ortodoncia (Daniel et al., 2013).

Teleortodoncia es la entrega de información de salud y atención a través de distancias utilizando tecnología de la información y telecomunicaciones. La teleortodoncia abarca diagnóstico, tratamiento, monitoreo, prevención y educación continua de proveedores y consumidores e investigación. La ATDA ha llevado a cabo una revisión exhaustiva y un análisis del tratamiento del alineador facilitado por la teleortodoncia (American Teledentistry Association, 2019).

Los datos de estudio sugieren que el tratamiento de teleortodoncia con alineadores transparentes es clínicamente efectivo en la corrección de problemas de alineación de incisivos maxilares y mandibulares (apiñamiento o espacios) de menos de $6 \mathrm{~mm}$ (Ackerman, 2019).

\section{Teleodontología en cirugía oral y/o maxilofacial}

La teleodontología podría ser una forma de mejorar el acceso a la atención especializada de ciru- 
MORÓN, A. M. La teleodontología una herramienta fundamental en tiempos de pandemia y post COVID -19, su utilidad en las diferentes especialidades odontológicas. Int. J. Odontostomat., 15(1):43-50, 2021.

gía oral y/o maxilofacial. Los teléfonos inteligentes proporcionan un acceso rápido y claro a las imágenes digitales enviadas por correo electrónico y permiten al cirujano oral y/o maxilofacial movilidad libre, no restringido por las limitaciones de una computadora personal de escritorio (Estai et al., 2016a,b; Birur et al., 2018; Giudice et al., 2020).

Por medio de imágenes y radiografías orales se ha detectado cáncer oral y patologías orales que puede beneficiarse la teleodontología como diagnósticos y no en términos de procedimientos como un medio para monitorear las condiciones postoperatorias y después de los tratamientos quirúrgicos inmediatos, el contacto con especialistas permite registrar un puntaje de dolor durante las evaluaciones postoperatorias sin la necesidad ir a un consultorio dental. Gracias a la colección de fotos, se puede analizar el sitio quirúrgico, la higiene bucal y la mejora de las funciones orales (Praveen et al., 2018; Haron et al., 2020).

La ATDA ha revelado que la barrera como lo es acceder a la atención de personas mayores es el costo. Otras barreras importantes notables incluyen dificultad para encontrar un odontólogo, ubicación, horarios. Para reducir o eliminar estas barreras el uso de la teleodontología se ha implementado de manera exitosa para la atención de adultos mayores.

Los nuevos enfoques de la teleodontología ayudarán a los odontólogos a orientar a los pacientes sin aumentar el riesgo de infección cruzada. En la actualidad por la pandemia del COVID-19, el uso de la teleodontología va enfocado hacia lograr el mejor diagnóstico posible para poder brindar orientación (Scarlette, 2020).

Actualmente existen dispositivos de alta tecnología como el "Mouth Watch," "Dental Monitoring", "Smile Mater "que nos ayudan a navegar frente los desafíos de COVID-19 y más allá. Con una probada plataforma de teledeodontologia en la nube enfocada en conectarse con los pacientes y su equipo de atención, virtualmente.

\section{Mouth Watch}

La teleodontología de Mouth Watch tiene la capacidad de comunicación de transmisión en vivo, junto con la cámara intraoral Mouth Watch, se puede conectar directamente a un computador portátil. El software basado en la nube permite que toda la documentación del paciente cargada previamente se inte- gre perfectamente con la conexión segura de transmisión en vivo, junto con cualquier nota del paciente (Heinrichs et al., 2019; Herman, 2020).

\section{Dental Monitoring}

Dental Monitoring afianza su posición de liderazgo en teleodontología basada en inteligencia artificial (IA) tras la adquisición de Loum, una empresa emergente en tecnología sanitaria de Europa.

Dental Monitoring adquirió Loum, "el asistente digital para pacientes y profesionales de la atención de la salud". Fundada en 2017, el objetivo de Loum es garantizar que los pacientes perciban la atención de salud que reciben en forma positiva, no como una carga. Sus desarrollos innovadores, en particular una aplicación diseñada para mejorar la experiencia de los pacientes, complementa el conjunto en expansión de herramientas basadas en inteligencia artificial (IA) de Dental Monitoring. La aplicación implementa un sistema dinámico y agradable que facilita la relación entre el odontólogo y el paciente, a la vez que permite que los pacientes se comprometan con el tratamiento completo.

Las soluciones de Dental Monitoring se encuentran diseñadas para asistir a los profesionales en salud dental durante el proceso de tratamiento de sus pacientes mediante:

- Una conexión más simple entre profesionales y pacientes.

- La facilidad en el seguimiento y una experiencia virtual más fluida durante el tratamiento.

- La mejora en la estabilidad del tratamiento y de los resultados con el transcurso del tiempo.

Dental Monitoring, con presencia en Europa, Estados Unidos y Asia y el Pacífico, cuenta con 5 oficinas en: París; Austin, Texas; Londres; Hong Kong y Sídney (Heinrichs et al.; Herman).

\section{CONCLUSIONES}

La odontología forma una parte importante del sistema de atención médica, que se ha visto gravemente comprometida durante la actual pandemia de COVID-19. La teleodontología no es un sustituto de la consulta presencial, y tiene objetivo principal es apoyar el sistema de salud durante la pandemia 
COVID-19. Siempre se requiera una consulta física después de la teleconsulta y telediagnóstico.

La teleodontología ha existido durante años, pero en este momento la tecnología está experimentando un renacimiento a medida que los proveedores de atención odontológica en todo el mundo están comenzando a comprender rápidamente su valor. También ofrece nuevas oportunidades para la educación dental al proporcionar a los profesionales un acceso fácil a consultas y al ayudarles a llevar a cabo programas de educación y educación dental continua.

La teleodontología mejora la calidad de la atención facilitando información mejor y oportuna a la odontología para una mejor toma de decisiones y mejor comunicación entre Odontólogos y pacientes. La integración de la tecnología en el entorno educativo odontológico y de la salud presenta retos no sólo tecnológicos sino humanos. Por otra parte, los beneficios son claros y a nuestro entender, es evidente que las escuelas y facultades de odontología se beneficiarán al invertir recursos en el desarrollo de las tecnologías que fortalezca sus actividades académicas tanto para profesionales como para estudiantes y pacientes.

MORÓN, A. M. Teledentistry a fundamental tool in times pandemic and Post COVID-19, its usefulness in different dental specialties. Int. J. Odontostomat., 15(1):43-50, 2021.

ABSTRACT:Coronavirus infection has been considered by the WHO (World Health Organization) as a global pandemic. Since its origin, it has posed a threat to global health, not so much because of its symptoms, but also because of its rapid spread and contagion and the respiratory complications that they produce in patients with other chronic underlying conditions. Telehealth has caused prominence in this time of COVID-19 since many face-to-face consultations have been closed to avoid contagion and virtuality has been preferred. Teledentistry has not been the exception, facilitating the consultation of patients, specialists and / or dental care providers at a distance to provide dental services, especially diagnostic and preventive optimized in the physical absence of the patient. This literature review article aims to describe the use of teledentistry as a tool in times of COVID-19, its application and use in different dental specialties.

KEY WORDS: COVID-19, Technology, Teledentistry.

\section{REFERENCIAS BIBLIOGRAFICAS}

Ackerman, M. B. Teleorthodontic treatment with clear aligners: An analysis of outcome in treatment supervised by general practitioners versus orthodontic specialists. J. Dent. Res. Rep., 2, 2019. DOI: https://www.doi.org/10.15761/JDRR.1000114
Almazrooaa, S. A.; Mansour, G. A.; Alhameda, S. A.; Ali, S. A.; Akeel, S. K.; Alhindi, N. A.; Felemban, O. M.; Mawardi, H. H. \& Binmadi, N. O. The application of teledentistry for Saudi patients' care: A national survey study. J. Dent. Sci., 2020. DOI: https:// www.doi.org/10.1016/j.jds.2020.04.014

American Dental Association. Teledentistry. Comprehensive ADA Policy Statement on Teledentistry. Chicago, American Dental Association, 2019. Disponible en: https:// www.americanteledentistry.org/wp-content/uploads/2019/11/ 2015-Teledentistry-Policy.pdf

American Teledentistry Association (ATDA). Teledental Practice and Teledental Encounters: An American Association of Teledentistry Position Paper. Website. American Teledentistry Association, 2018. Disponible en: https://www.americanteledentistry.org/wp$\mathrm{c} \circ \mathrm{nte} \mathrm{nt} / \mathrm{u} \mathrm{p} / \mathrm{o}$ a d s / $2018 / 10 /$ ATDA_TeledentalPracticePositionPaper.pdf

American Teledentistry Association (ATDA). Teleorthodontics and Clear Aligner Treatment: An American Teledentistry Association Position Paper. Website. American Teledentistry Association, 2019. Disponible en: http://www.americanteledentistry.org/wpcontent/uploads/2019/12/ATDA-POSITION-PAPER_Finalcopy1.pdf

Asociación Latinoamericana de Odontopediatría (ALOP). Teleodontología: aplicación a la odontopediatría durante la pandemia COVID-19. Rev. Odontopediatría Latinoam., 10(2), 2020. Disponible en: https://www.revistaodontopediatria.org/ediciones/2020/2/art-3/

Avula, $\mathrm{H}$. Tele-periodontics - Oral health care at a grass root level. $J$. Indian Soc. Periodontol., 19(5):589-92, 2015.

Birur, N. P.; Patrick, S.; Bajaj, S.; Raghavan, S.; Suresh, A.; Sunny, S. P.; Chigurupati, R.; Wilder-Smith, P.; Gurushanth, K.; Gurudath, S.; et al. A novel mobile health approach to early diagnosis of oral cancer. J. Contemp. Dent. Pract., 19(9):1122-8, 2018.

Bradley, M.; Black, P.; Noble, S.; Thompson, R. \& Lamey, P. J. Application of teledentistry in oral medicine in a community dental service. N. Ireland. Br. Dent. J., 209(8):399-404, 2010.

Cáceres-Méndez, E. A.; Castro-Díaz, S. M.; Gómez-Restrepo, C. \& Puyana, J. C. Telemedicina: historia, aplicaciones y nuevas herramientas en el aprendizaje. Univ. Med., 52(1):11-35, 2011.

Cardwell, J. Asynchronous teledentistry: It's like a DVR for patient care. Website. DentalEconomics, 2020. Disponible en: https:// www.dentaleconomics.com/science-tech/oral-medicineanesthetics-and-the-oral-systemic-connection/article/14073055/ asynchronous-teledentistry-its-like-a-dvr-for-patient-care

Cruvinel, T.; Aguirre, P. E. A.; Lotto, M.; Oliveira, T. M.; Rios, D. \& Cruvinel, A. F: P. Digital behavior surveillance: Monitoring dental caries and toothache interests of Google users from developing countries. Oral Dis., 25(1):339-47, 2019.

Daniel, S. J.; Wu, L. \& Kumar, S. Teledentistry: a systematic review of clinical outcomes, utilization and costs. J. Dent. Hyg., 87(6):345-52, 2013.

Estai, M.; Bunt, S.; Kanagasingam, Y. \& Tennat, M. Cost savings from a teledentistry model for school dental screening: an Australian health system perspective. Aust. Health Rev., 42(5):482-90, 2018.

Estai, M.; Bunt, S.; Kanagasingam, Y.; Kruger, E. \& Tennant, M. Diagnostic accuracy of teledentistry in the detection of dental caries: a systematic review. J. Evid. Based Dent. Pract. 16(3):161-72, 2016a.

Estai, M.; Kanagasingam, Y.; Huang, B.; Checker, H.; Steele, L.; Kruger, E. \& Tennant, M. The efficacy of remote screening for dental caries by mid-level dental providers using a mobile teledentistry model. Community Dent. Oral Epidemiol., 44(5):435$41,2016 b$.

Ghai, S. Teledentistry during COVID-19 pandemic. Diabetes Metab. Syndr., 14(5):933-5, 2020. 
MORÓN, A. M. La teleodontología una herramienta fundamental en tiempos de pandemia y post COVID -19, su utilidad en las diferentes especialidades odontológicas. Int. J. Odontostomat., 15(1):43-50, 2021

Giudice, A.; Barone, S.; Muraca, D.; Averta, F.; Diodati, F.; Antonelli, A. \& Fortunato, L. Can teledentistry improve the monitoring of patients during the Covid-19 dissemination? A descriptive pilot study. Int. J. Environ. Res. PublicHealth, 17(10):3399, 2020.

Haron, N.; Zain, R. B.; Ramanathan, A.; Abraham, M. T.; Liew, C. S.; Ng, K. G.; Cheng, L. C.; Husin, R. B.; Chong, S. M. Y.; Thangavalu, L. A. P.; et al. $\mathrm{m}$-Health for early detection of oral cancer in low- and middle-income countries. Telemed. J. E Health, 26(3):278-85, 2020.

Heinrichs, J. How Using Teledentistry to Conduct Free Screenings Resulted in $60 \%$ New-Patient Conversions and Helped Me Sell My Practice. Website. DentalEconomics.com, 2019. Disponible en: https:// www.dentaleconomics.com/practice/systems/article/14069052/howusing-teledentistry-to-conduct-free-screenings-resulted-in-60newpatient-conversions-and-helped-me-sell-my-practice

Herman, B. Teledentistry: How visual treatment planning goes virtual. Website. DentalEconomics.com, 2020, Disponible en: https:/ /www.dentaleconomics.com/practice/patient-communicationand-patient-financing/article/14173734/teledentistry-how-visualtreatment-planning-goes-virtual

Jain, M. Teledentistry. Current Therapy in Endodontics. Dubai, John Wiley \& Sons, 2016.

Márquez, V. J. R. Teleconsulta en la pandemia por Coronavirus: desafíos para la telemedicina pos-COVID-19. Rev. Colomb. Gastroenterol., 35(Supl. 1):5-16, 2020.

Meng, L.; Huan, F. \& Bian, Z. Coronavirus Disease 2019 (COVID19): emerging and future challenges for dental and oral medicine. J. Dent. Res., 99(5):481-7, 2020.

Petruzzi, M. \& De Benedittis, M. WhatsApp: a telemedicine platform for facilitating remote oral medicine consultation and improving clinical examinations. Oral Surg. Oral Med. Oral Pathol. Oral Radiol., 121(3):248-54, 2016.

Purohit, B. M.; Singh, A. \& Dwivedi, A. Utilization of teledentistry as a tool to screen for dental caries among 12-year-old school children in a rural region of India. J. Public Health Dent., 77(2):174-80, 2017.

Quispe-Juli, C.; Vela-Anton, P.; Meza-Rodriguez, M. \& MoquillazaAlcántara, V. COVID-19: Una pandemia en la era de la salud digital. Preprints ScIELO, 2020 Disponible en: https:// webcache.googleusercontent.com/search?q=cache:LHLuvz0TFkJ:https://preprints.scielo.org/index.php/scielo/preprint/ download $/ 164 / 195 / 183+\& c d=1 \&$ hl=es-419\&ct=clnk\&gl=cl

Rodríguez Díaz, W. D. C. \& Cabardillo, L. D. A. Pasado, presente y futuro de la teleodontología: un nuevo reto. Puente Rev. Cient., 94-8, 2014.

Rojas Maldonado, M. C.; Cardona Gómez, L. M. \& Fernández Herrera, C. A. La Tele-Odontología. Una Nueva Herramienta para Diferenciar Nuestras Instituciones Prestadoras de Salud (IPS). Tesis de Grado. Medellín, Universidad CES, 2015. Disponible en: http://hdl.handle.net/10946/2733

Scarlette, M. Teledentistry, A Solution for Our New Coronavirus Reality. Website. DentalEconomics.COM, 2020. Disponible en: https://www.dentaleconomics.com/practice/article/14175781/ teledentistry-a-solution-for-dentistrys-new-coronavirus-reality

Sigua-Rodríguez, E. A.; Bernal-Pérez, J. L.; Lanata-Flores, A. G.; Sánchez-Romero, C.; Rodríguez-Chessa, J.; Haidar, Z. S.; Olate, S. \& Iwaki-Filho, L. COVID-19 and dentistry: a review of recommendations and perspectives for Latin America. Int. J. Odontostomat., 14(3):299-309, 2020.

Telles-Araujo, G. T.; Garcia Caminha, R. D.; Samaan Kallás, M. \& da Silva Santos, P. S. Teledentistry support in COVID-19 oral care. Clinics, 75:e2030, 2020.

Turner, M. 5 Tips On How To Incorporate Teledentistry Services Into Your Dental Practice Right Now. Website. DentalEconomics.com, 2020. Disponible en: https://www.dentaleconomics.com/print/ content/14177520
Dirección para correspondencia:

Michelle Morón Araújo

Odontóloga

Pontificia Universidad Javeriana Bogotá

COLOMBIA

Email: michellemoronaraujo@gmail.com 